\title{
An improved artificial potential field algorithm based on nonuniform cell decomposition
}

\author{
Yanyi Yang ${ }^{1,}$, Yingming Zhang ${ }^{2, b}$ and Xingchen $\mathrm{Li}^{1, \mathrm{c}}$ \\ ${ }^{1}$ National NC System Engineering Research Center, Huazhong University of Science and \\ Technology, Wuhan 430074, PR China \\ ${ }^{2}$ State key laboratory of materials processing and Die \& Mold Technology, Huazhong University of \\ Science and Technology, Wuhan 430074, PR China \\ ayangyanyi@163.com, bzhangyingming@hust.edu.cn, Clixingchen@hust.edu.cn
}

Keywords: path planning; quadtree algorithm; artificial potential field algorithm

\begin{abstract}
Path planning of mobile robot is a complex issue involves computing and optimizing a collision-free path between initial position and destination, which requires the method reliable and efficient. This paper introduces an improved artificial potential field (APF) based on nonuniform decomposition method. The nonuniform decomposition can not only expand the range of robot's workspace and increase the possibilities of feasible paths existing, but also promote the efficiency of path planning. The improved APF algorithm solves some key problems of traditional APF. At last, the result of simulation verifies the effectiveness of this method.
\end{abstract}

\section{Introduction}

The path planning is a crucial issue in research field of mobile robot which has been researched for many years. The main purpose of planning is to search the optimum path satisfying minimum distance and maximum safety from initial position to destination in given environment with obstacles. Under some situations of environmental information completely known, path planning involves two basic steps: environment modelling and path searching.

In environment modelling, there is a common method termed Grid algorithm [1]. The characteristic exerted which makes Grid algorithm attractive is it can be built easily and fix the position of mobile robot exactly. However, when environment is complex, these methods will be time-consuming and tedious. In path searching, there are some algorithm [2-5] realizing the searching process. Among above algorithm, the APF algorithm is more popular due to its simpler algorithm and more reliable result. Nevertheless, there are two major disadvantages of APF: (1) Robot is prone to fall into local minimum point. (2) Robot can't reach the goal point successfully.

To solve these problems above described, this paper introduces an inproved APF algorithm based nonuniform cell decomposition to realize path planning in high effciency.

Nonuniform cell decomposition. This method can partition two-dimension environment nonuniformly by quadtree algorithm until the specific criterion is met. Some factors including less computer storage space, higher computation efficiency, and little wasted space of environment are considered to determine the minimum size of cell subdivided. Compared with uniform decomposition based on grid algorithm, the nonuniform decomposition can lose connectivity graph, which means a cell could not find the neighbor cells surrounding it in the process of nonuniform decomposition. A traverse operation will be implemented for all free cells to find their neighbor free cells. The characteristic of two adjacent cells is they share the common edge or vertex. Based on this characteristic, we can obtain a series of neighbor free cells of the current cell and build the structure of connectivity graph for them.

More descriptions of nonuniform cell decomposition based on quadtree algorithm [6] are illustrated as follow: 
(1) $S$ (a collection of cells) is initialized as the collection including only a cell which is the entire environment. $O$ (a collection of obstacle cells) and $F$ (a collection of free cells) are initialized as two empty collections.

(2) Each cell from $S$ is split into two parts along X, Y direction respectively, so we can get four new cells from the original cell. According to our definition, the new cells can be classified three types: free cell, blend cell, and obstacle cell, which respectively indicate there is no obstacles in the cell, the cell is filled with obstacles partially, and the cell is filled with obstacles completely.

(3) If new cell is free cell, the cell won't be subdivided any longer, and it will be added in collection $F$. If the new cell is blend cell and the size is smaller than the specified minimum size $a$, the cell is treated as obstacle cell which will be stopped to subdivide, then it will be added in collection $O$. If the size of new blend cell is larger than $a$, the cell will be returned as an input in (2) until there are only free cells and obstacle free defined in the environment.

(4) For each element in collection $F$, a searching operation for neighbor cells among free cells will be implemented. If there are some free cells which share common edge or vertex with the current free cell, these free cells are considered as the neighbor cells and need to be built the connectivity graph with the current free cell.

By comparing the experiment results of two methods in Fig. 1(a) and in Fig. 1(b), we can get the Nonuniform decomposition algorithm need generate less cells in the environment which reduces computer storage space and promotes the efficiency of path planning algorithm to some extent. The concrete comparison can be seen in Table 1 .

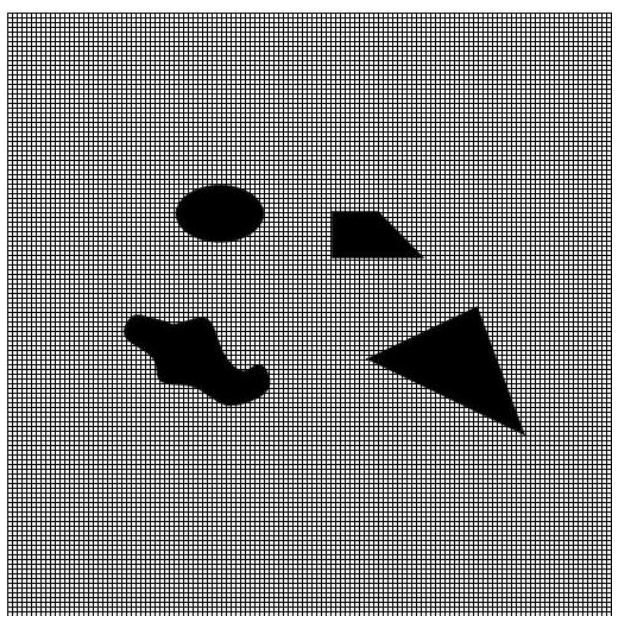

(a)

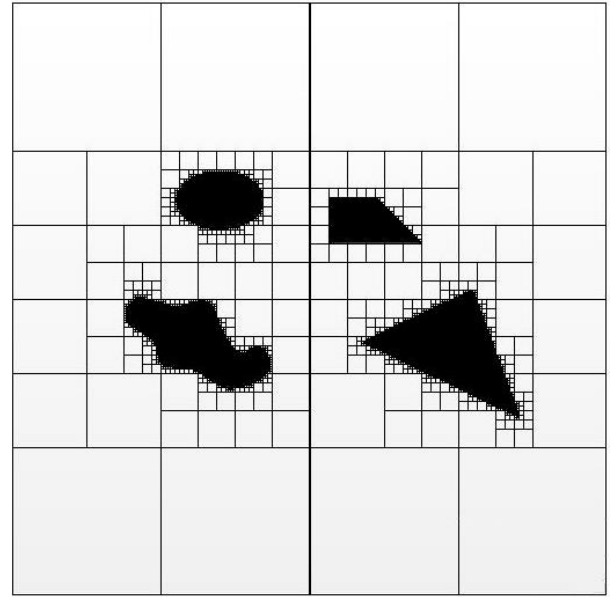

(b)

Fig. 1. (a) The environment modelling method based on Grid algorithm (b) The environment modelling method based on Nonuniform decomposition algorithm

Table 1. The comparison of two environment modelling methods.

\begin{tabular}{cccc}
\hline Method & Free grid & Obstacle grid & Total grid \\
\hline Grid algorithm & 6424 & 632 & 7056 \\
Nonuniform decomposition & 752 & 168 & 920 \\
\hline
\end{tabular}

Improved APF algorithm. The traditional APF is generated with destination's attractive field and the obstacle's repulsive field. Therefore, this method can be written as:

$$
U_{\text {total }}=U_{\text {att }}+U_{\text {rep }} \text {. }
$$

Where $U_{a t t}$ and $U_{r e p}$ are the attractive and repulsive fields in which the robot locates. 
The resultant force of robot in the potential field is:

$$
F_{\text {total }}=F_{\text {att }}+F_{\text {rep }}
$$

Where $F_{a t t}$ and $F_{r e p}$ are the gravitational force and the repulsive force which produce the combine effects on the robot.

The attractive field function $U_{a t t}$ can be defined as:

$$
U_{\text {att }}=\frac{1}{2} k_{a t t} \rho_{g}^{2}
$$

Here $k_{a t t}$ is a constant scaling factor about attractive field, $\rho_{g}=\left\|X-X_{d}\right\|$ is the Euclidean distance between initial position and destination. $X$ and $X_{d}$ are the coordinates where current point and target point are located. The gravitational force is obtained by differentiating the attractive field function $U_{a t t}$ and the equation is:

$$
F_{a t t}(X)=-\nabla U_{a t t}(X)=-k\left(X-X_{d}\right)
$$

The $U_{\text {rep }}$ can be defined as:

$$
U_{\text {rep }}(X)=\left\{\begin{array}{cc}
-\frac{1}{2} k_{\text {rep }}\left(\frac{1}{\rho(X)}-\frac{1}{\rho_{o}}\right)^{2}, & \rho(X) \leq \rho_{o} . \\
0 & , \quad \rho(X) \geq \rho_{o}
\end{array}\right.
$$

Here $k_{r e p}$ is a constant scaling factor about repulsive field. $\rho(X)$ is the distance between obstacles and robot. $\rho_{o}$ is the limit distance of the repulsive field which is influenced by the obstacle. The gravitational force can be written as:

$$
F_{r e p}(X)=\left\{\begin{array}{cc}
k_{r e p}\left(\frac{1}{\rho(X)}-\frac{1}{\rho_{o}}\right) \frac{1}{\rho(X)^{2}} \frac{\partial \rho}{\partial X} & \rho(X) \leq \rho_{o} \\
0 & , \quad \rho(X) \geq \rho_{o}
\end{array} .\right.
$$

In traditional APF algorithm, when the destination is very close to obstacles, robot could not reach the destination. When mobile robot is subjected to attraction and repulsion with the same size and the opposite direction, the total potential force acting on robot is zero, which will lead robot to be trapped in local minima $[6,7]$.

To avoid these problems described above and improve efficiency of path searching, an improved APF algorithm based on cells is proposed. In this method, instead of calculating the real-time resultant force which guides robot toward destination, we exploit to calculate resultant force of neighbor cells surrounding the cell where the initial point is located and select one cell containing maximum resultant force and the minimum deflection angle with the direction toward destination as the target cell that needs to reach in next step. The details about this algorithm can be seen in Fig. 2 . The robot in the start position need to select one of some neighbor cells as the next position. Just the cell satisfying maximum magnitude of $F_{\text {total }} \cdot \vec{e}$ among all neighbor free cells will be selected. 


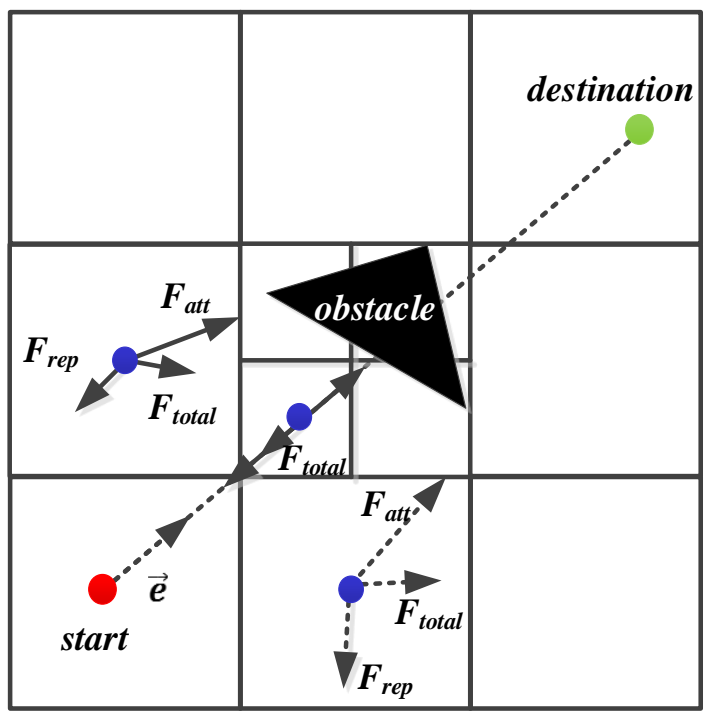

Fig. 2. The diagram of the improved APF algorithm.

The algorithm of improved APF is illustrated as follows:

(1) Calculate the unit vector $\vec{e}$ from initial position to destination,

(2) Get neighbor cells of the cell where the initial point is located.

(3) FOR each cell of neighbor cells,

(4) If the neighbor cell is the cell where the destination is located, go to (11).

(5) Else, go to (7).

(6) Calculate the resultant force $F_{\text {total }}$ by using Eq.2

(7) Calculate the magnitude of $F_{\text {total }} \cdot \vec{e}$.

(8) End FOR

(9) Get the target cell which has maximum magnitude of $F_{\text {total }} \cdot \vec{e}$.

(10) Reach the target cell.

(11) If the target is same with cell located destination, go to (13).

(12) Else, the target is set as initial position, then go to (1)

(13) END

Path planning experiment. The simulation software has been implementation in $\mathrm{C}++$ code. The result proves the feasibility and effectiveness of the algorithm in Fig. 3. The green line represents the path obtained finally. Some black blocks represent obstacles in the environment. The red dot indicates the robot. The effect is obvious that the robot can go through all the obstacles safely and the path is collision-free. 


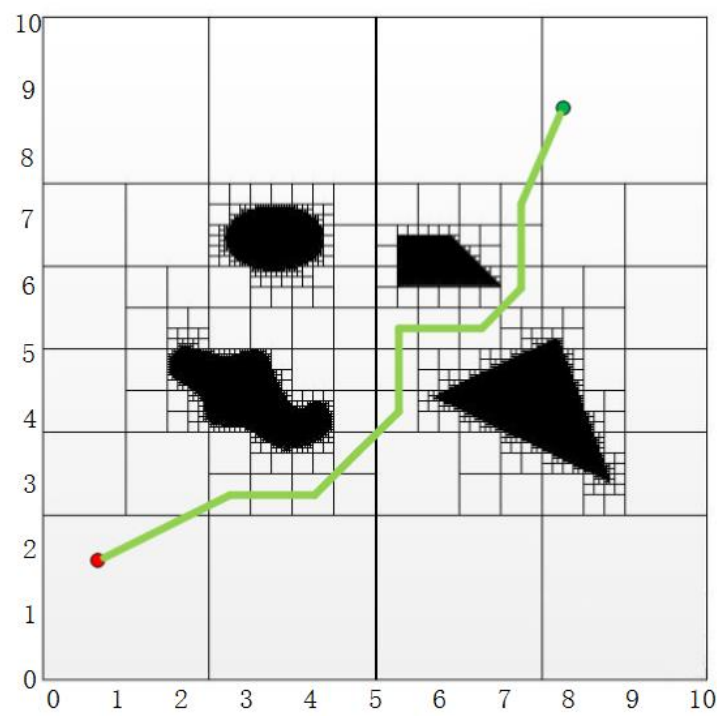

Fig. 3. Simulation results with this path planning method proposed

\section{Conclusions}

This paper developed path planning method under known environment. The path planning method can be divided in two steps. In the first step, a nonuniform cell decomposition by using quadtree is traduced to promote the environment modelling as can be seen from simulation result. In the second step, an improved artificial potential field is introduced to solve some deficiency existed in traditional APF. The experiment is carried out to validate the method proposed.

\section{Acknowledgements}

This work was financially supported by the Graduates' Innovation Fund, Huazhong University of Science and Technology (5003100009).

\section{References}

[1] G. Zhou, "A Fast Algorithm Based on Grids for Mobile Robot Path Planning," Computer Engineering \& Applications, vol. 42, pp. 197-199, 2006.

[2] Z. Zhang and Z. Zhao, "A Multiple Mobile Robots Path planning Algorithm Based on A-star and Dijkstra Algorithm," International Journal of Smart Home, vol. 8, pp. 75-86, 2014.

[3] G. Nagib and W. Gharieb, "Path planning for a mobile robot using genetic algorithms," in International Conference on Electrical, Electronic and Computer Engineering, 2004, pp. 185-189.

[4] S. H. Chia, K. L. Su, J. H. Guo, and C. Y. Chung, "Ant Colony System Based Mobile Robot Path Planning," in Fourth International Conference on Genetic and Evolutionary Computing, 2011, pp. 210-213.

[5] H. Noborio, T. Naniwa, and S. Arimoto, "A quadtree - based path - planning algorithm for a mobile robot," Journal of Field Robotics, vol. 7, pp. 555-574, 2010.

[6] L. Chen, C. Liu, H. Shi, and B. Gao, "New Robot Planning Algorithm Based on Improved Artificial Potential Field," in International Conference on Instrumentation, 2014, pp. 228-232.

[7] G. Li, A. Yamashita, H. Asama, and Y. Tamura, "An efficient improved artificial potential field based regression search method for robot path planning," in International Conference on Mechatronics and Automation, 2012, pp. 1227-1232. 